\title{
NONLINEAR TRANSFORMATIONS OF VOLTERRA TYPE IN WIENER SPACE $\left.{ }^{1}\right)$
}

\author{
BY \\ R. H. CAMERON AND R. E. FAGEN
}

1. Introduction. In a previous paper by Cameron and Martin [2] $\left.{ }^{2}\right)$, the behavior of Wiener integrals under transformations of the form

$$
T: \quad y(t)=x(t)+\Lambda(x \mid t)
$$

was studied, and theorems giving a formula for the transformation of Wiener integrals (in particular, Theorem A below) were obtained, where $\Lambda(x \mid t)$ was a (generally nonlinear) functional depending on the function $x$ and the number $t$ and satisfying certain smoothness conditions. (The number $t$ ranges over the interval $I: 0 \leqq t \leqq 1$, and the function $x$ ranges over a measurable set $\Gamma$ of the space $C$ of continuous functions on $I$ which vanish at $t=0$.) The primary smoothness condition on $\boldsymbol{\Lambda}$ was that it be of smooth variation in a Hilbert neighborhood of each point of the set $\Gamma$, according to the following definition.

Definition 1. A functional $\Lambda(x \mid t)$ defined on $S \otimes I$, where $S$ is a convex subset of $C$ open in the uniform topology, will be said to be "of smooth variation" if its first variation

$$
\delta \Lambda(x|t| y) \equiv \frac{\partial}{\partial v} \Lambda(x+v y \mid t)_{v=0}
$$

exists for all $(x, t, y)$ in $S \otimes I \otimes C$ and is representable in the form

$$
\delta \Lambda(x|t| y)=\int_{0}^{1} K(x \mid t, s) y(s) d s, \quad(x, t, y) \in S \otimes I \otimes C ;
$$

where $K(x \mid t, s)$ is continuous in $(x, t, s)$ throughout $S \otimes I^{2}$ (continuity in $x$ being understood in the uniform topology). The functional $K(x \mid t, s)$ will be called "the kernel of the variation of $\Lambda(x \mid t)$. " It is of course the classical Volterra derivative of the functional $\Lambda$. (See [3, p. 49].)

It turns out, however, that the functionals $\Lambda$ which are of the "Volterra type," i.e., which depend on integrals with variable upper limit, do not usually satisfy the condition, though they may satisfy all but the continuity condition on $K$. Thus, if

Presented to the Society, April 25, 1953; received by the editors October 30, 1952.

(') Research sponsored by the Office of Ordnance Research, U.S. Army, under contract DA-11-022-ORD-489.

(2) Numbers in brackets refer to the references at the end of the paper. 


$$
\Lambda(x \mid t)=\int_{0}^{t} f(t, s, x(s)) d s,
$$

where $f(t, s, u)$ and $f_{u}(t, s, u)$ are continuous in $I^{2} \otimes(-\infty, \infty)$, we have

$$
\delta \Lambda(x \mid t, y)=\int_{0}^{t} f_{u}(t, s, x(s)) y(s) d s,
$$

which can be written in the form (1.3) if

$$
K(x \mid t, s)= \begin{cases}f_{u}(t, s, x(s)) & \text { if } 0 \leqq s<t \leqq 1 \\ 0 & \text { if } 0 \leqq t<s \leqq 1\end{cases}
$$

But this shows that (1.4) cannot be of smooth variation unless $f$ satisfies the highly artificial condition

$$
f_{u}(t, t, x(t))=0
$$

for $t \in I$ and each $x$ in $S$.

In order to be able to handle functionals of the form (1.4) which do not satisfy (1.6), we shall consider in this paper functionals of semi-smooth variation, defined as follows.

Definition 2. A functional $\Lambda(x \mid t)$ defined on $S \otimes I$, where $S$ is a convex subset of $C$ open in the uniform topology, will be said to be "of semi-smooth variation" if its first variation (1.2) exists for all $(x, t, y)$ in $S \otimes I \otimes C$ and is representable in the form (1.3), where

$$
K(x \mid t, s)= \begin{cases}K^{1}(x \mid t, s) & \text { when } 0 \leqq t<s \leqq 1 \\ 2^{-1} K^{1}(x \mid t, s)+2^{-1} K^{2}(x \mid t, s) & \text { when } 0 \leqq s=t \leqq 1 \\ K^{2}(x \mid t, s) & \text { when } 0 \leqq s<t \leqq 1\end{cases}
$$

and $K^{1}$ and $K^{2}$ are continuous in $(x, t, s)$ throughout $S \otimes\{0 \leqq t \leqq s \leqq 1\}$ and $S \otimes\{0 \leqq s \leqq t \leqq 1\}$ respectively (continuity in $x$ meaning continuity in the uniform topology). Again, $K$ is "the kernel of the variation of $\Lambda$. "

It is clear that the functional (1.4) satisfies this definition if $S$ is a uniform sphere and if in (1.5) we put $K(x \mid t, t)=2^{-1} f_{u}(t, t, x(t))$; and this illustrates the fact that the class of semi-smooth functionals is considerably wider than the class of smooth functionals. (We shall later see that the convention in (1.7) which requires the value on the diagonal to be the average of the right and left limits is not merely arbitrary, but actually plays an important role in simplifying our transformation formula. )

In this paper we shall so extend the transformation formula of [2] that it can be applied to transformations involving functionals of semi-smooth variation. Moreover our results will be more general from another standpoint which is likely to be important in applications; namely, we shall require only that our conditions on $\Lambda(x \mid t)$ and $K(x \mid t, s)$ shall hold in a uniform neighborhood 
of each point of our region under consideration instead of a Hilbert neighborhood. It is interesting that the very methods of attack we use in dealing with the primary generalization to functionals of semi-smooth variation also yield this relaxation of the topological requirements. Since the statement of the earlier theorem involved both topologies in an essential way, the present theorem is not only more general but also simpler and less confusing. Finally, it will be noted that this same method of attack permits us to make less restrictive requirements on the kernel $K$.

TERMINOLOGY AND NOTATION. It shall hereafter be understood that all topological terms are to be interpreted in the uniform topology for $C$ unless otherwise specified. The Hilbert and uniform norms will be denoted respectively by $\|||$ and \|\|$\|$. Moreover Stieltjes integrals are to be interpreted as RiemannStieltjes integrals unless otherwise specified. Finally, derivatives will be denoted by the differential notation or by subscripts-never by primes. Thus, in this paper $x(t)$ and $x^{\prime}(t)$ are simply two unrelated functions. The same applies to sets, where $S$ and $S^{\prime}$ may be any two unrelated sets.

In order to obtain a result of any sort under the new conditions, it has been found necessary to introduce a new concept, which is in a certain sense a modification of the Stieltjes integral.

Definition 3. Let $F(x \mid t)$ be a functional defined for $x$ and $t$ on $S \otimes I$ (where $S$ is an open subset of $C$ ) and integrable in t for each $x$. Let $x_{\epsilon}$ be the lower $\epsilon$-smoothing of $x$ :

$$
x_{\epsilon}(t)=\frac{1}{\epsilon} \int_{t-\epsilon}^{t} x(s) d s
$$$$
[x(t)=0 \text { if } t<0]
$$

and let $F_{\epsilon}(x \mid t)$ be the upper smoothing of $F\left(x_{\mathrm{e}} \mid t\right)$ :

$$
F_{\epsilon}(x \mid t)=\frac{1}{\epsilon} \int_{t}^{t+\epsilon} F\left(x_{\epsilon} \mid s\right) d s
$$

(where $F(x \mid s)$ is understood to be constant for $s$ on $1 \leqq s<\infty$ and fixed $x$ ). Then we define "the functional principal value of the Stieltjes integral of $F$ with respect to $x(t)$ " to be the following limit, if it exists (as a finite number):

$$
\int_{0}^{1} F(x \mid t) d^{*} x(t)=\lim _{\epsilon \rightarrow 0^{+}} \int_{0}^{1} F_{\epsilon}(x \mid t) d x(t) .
$$

This definition of course leads to the question of existence and interpretation. The following two theorems which we shall prove in $\$ 2$ supply information sufficient for our purposes in the discussions to follow. Theorem 1 deals with the general $F(x \mid t)$ satisfying rather strong smoothness conditions, while Theorem 2 deals with a more specialized $F(x \mid t)$ (a type arising in connection with the application of the transformation theory to (1.4)), under less restrictive smoothness conditions. 
ThEOREM 1. Let $F(x \mid t)$ be defined on $S \otimes I$, where $S$ is a neighborhood of a fixed point $x_{0} \in C$, let $F(x \mid t)$ be integrable in $t$ on I for each $x \in S$, and let $F(x \mid 1)$ be continuous in $x$ at $x=x_{0}$. Then we have

$$
\int_{0}^{1} F\left(x_{0} \mid t\right) d^{*} x_{0}(t)=\int_{0}^{1} F\left(x_{0} \mid t\right) d x_{0}(t),
$$

provided that $\int_{0}^{1} F(x \mid t) d x(t)$ exists for $x \in S$ and is continuous at $x=x_{0}$. In particular, this condition will be fulfilled if $F(x \mid t)$ is of bounded variation on $I$ for each $x \in S$ and $\operatorname{var}_{t \in I}\left[F(x \mid t)-F\left(x_{0} \mid t\right)\right] \rightarrow 0$ as $x \rightarrow x_{0}$.

THEOREM 2. If $F(x \mid t)=f(t, x(t))$, where $f(t, u)$ and $\partial f(t, u) / \partial t$ are continuous in $(t, u)$ on $I \otimes(-\infty, \infty)$, then the functional principal value of the Stieltjes integral of $F$ with respect to $x(t)$ exists for each $x \in C$ and has the value

$$
\int_{0}^{1} F(x \mid t) d^{*} x(t)=g(1, x(1))-\int_{0}^{1} g_{t}(t, x(t)) d t,
$$

where

$$
g(t, u)=\int_{0}^{u} f(t, v) d v
$$

and

$$
g_{t}(t, u)=\partial g(t, u) / \partial t .
$$

We now state the most important results of this paper, which are two transformation theorems; Theorem 3 being a local theorem and Theorem 4 the corresponding nonlocalized theorem.

TheOREM 3. Let $S_{0}$ be a sphere in $C$ with center $x_{0}$ and let $\Lambda(x \mid t)$ be of semismooth variation in $S_{0} \otimes I$ with kernel $K(x \mid t, s)$. Let $\Lambda$ and $K$ satisfy the following seven conditions:

(1.14.1) $\Lambda(x \mid t) \in C$ when $x \in S_{0}$ and $\Lambda_{t}(x \mid t) \equiv \partial \Lambda(x \mid t) / \partial t$ exists and is continuous in $(x, t)$ on $S_{0} \otimes I$.

(1.14.2) $K(x \mid 0, s)=0$ for $(x, s) \in S_{0} \otimes(0,1]$.

(1.14.3) For each fixed $t \in I,\left\|K\left(x_{1} \mid t, \cdot\right)-K\left(x_{2} \mid t, \cdot\right)\right\| \rightarrow 0$ uniformly $x_{1}, x_{2} \in S_{0}$ as $\left\|\left|x_{1}-x_{2}\right|\right\| \rightarrow 0$.

(1.14.4) $K(x \mid t, s)$ is bounded for $x \in S_{0}$ and $s, t \in I$.

(1.14.5) $D\left(x_{0}\right) \neq 0$; where $D(x)$ denotes the Fredholm determinant

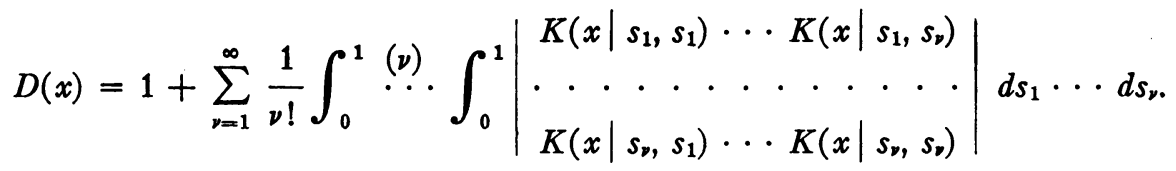

$$
\int_{0}^{1} \Lambda_{t}(x \mid t) d^{*} x(t) \text { exists for almost all } x \in S_{0} \text {. }
$$




$$
\int_{0}^{1}\left[\frac{\partial}{\partial t} \Lambda_{\epsilon}(x \mid t)\right] d x(t)
$$

is defined and bounded below in $(x, \epsilon)$

on $S_{0} \otimes\left(0, \epsilon_{0}\right)$ for some positive $\epsilon_{0}<1 / 2$.

Then there exists a sphere $S$ with center $x_{0}$ such that if $\Gamma$ is any Wiener measurable subset of $S$, its transform $T \Gamma$ by the transformation $T$ of (1.1) is Wiener measurable. Moreover if $F(y)$ is any Wiener measurable functional on $T \Gamma$ that makes either side of the following exist, both sides exist and they are equal:

$$
\begin{aligned}
\int_{T_{\Gamma}}^{W} F(y) d_{w} y=\int_{\Gamma}^{W} F(x+\Lambda(x \mid \cdot)) & \exp \left\{-2 \int_{0}^{1} \Lambda_{t}(x \mid t) d^{*} x(t)\right. \\
& \left.-\int_{0}^{1}\left[\Lambda_{t}(x \mid t)\right]^{2} d t\right\}|D(x)| d_{w} x .
\end{aligned}
$$

We call attention here to the fact that this formula holds by virtue of the convention that on the diagonal $s=t, K$ is the average of $K^{1}$ and $K^{2}$. The value of the determinant actually depends on the value of $K$ on the diagonal, and a different convention would render the formula (1.15) incorrect.

THEOREM 4. Let $\Gamma$ be any Wiener measurable subset of $C$, and assume that the transformation $T$ of (1.1) takes $\Gamma$ into $T \Gamma$ in a 1-to-1 manner and that $\Lambda$ is of semi-smooth variation in $S_{0} \otimes I$ for a neighborhood $S_{0}=S_{0}\left(x_{0}\right)$ of each point $x_{0}$ of $\Gamma$. Assume further that the conditions (1.14.1)-(1.14.7) hold in $S_{0}$ for each $x_{0} \in \Gamma$. Then $T \Gamma$ is also a Wiener measurable set; and if $F(y)$ is a Wiener measurable functional that makes either side of (1.15) exist, they both exist and are equal.

Our method of attack in proving these transformation theorems will be the following. We shall apply the results of the previous paper [2] to a family of "smoothed" transformations which we shall show satisfy all the hypotheses of Theorem $\mathrm{V}$ of that paper. By a limiting argument we shall show that this yields the desired formula locally under slightly more restricted conditions on $K(x \mid t, s)$. This result (Theorem 3a) then leads to a correspondingly restricted form of Theorem 4 (Theorem $4 \mathrm{a}$ ), by an argument identical with the one used in [2] to obtain its Theorem V from its Theorem IV. A second application of the "smoothing" argument leads from Theorem $4 \mathrm{a}$ to Theorem 3; and Theorem 4 follows from 3 in the same way that 4 a followed from $3 a$.

The authors wish to thank W. T. Martin for checking our results at an intermediate stage.

2. Functional principal values of Stieltjes integrals. We now proceed to establish Theorems 1 and 2.

Proof of Theorem 1. Let 


$$
G_{F}[x] \equiv \int_{0}^{1} F(x \mid t) d x(t)
$$

whenever the functional $F$ and the function $x$ are such that the RiemannStieltjes integral exists in the ordinary sense. Then it is clear that

$$
G_{F_{\epsilon}}[x]=\int_{0}^{1} F_{\epsilon}(x \mid t) d x(t)
$$

exists in some neighborhood of $x_{0}$, for $F_{\mathrm{e}}$ is the smoothing of $F\left(x_{\mathrm{e}} \mid t\right)$ and is hence absolutely continuous. Moreover, integrating by parts and using the fact that $F(x \mid s)=F(x \mid 1)$ on $1 \leqq s<\infty$, we have

$$
\begin{aligned}
G_{F \epsilon}[x]= & \frac{1}{\epsilon} \int_{0}^{1} \int_{t}^{t+\epsilon} F\left(x_{\epsilon} \mid s\right) d s d x(t)=\frac{1}{\epsilon} \int_{1}^{1+\epsilon} F\left(x_{\epsilon} \mid s\right) d s \cdot x(1) \\
& -\frac{1}{\epsilon} \int_{0}^{1} x(t)\left[F\left(x_{\epsilon} \mid t+\epsilon\right)-F\left(x_{\epsilon} \mid t\right)\right] d t \\
= & F\left(x_{\epsilon} \mid 1\right) x(1)-\frac{1}{\epsilon} \int_{\epsilon}^{1+\epsilon} x(t-\epsilon) F\left(x_{\epsilon} \mid t\right) d t+\frac{1}{\epsilon} \int_{0}^{1} x(t) F\left(x_{\epsilon} \mid t\right) d t \\
= & \frac{1}{\epsilon} \int_{0}^{1+\epsilon}[x(t)-x(t-\epsilon)] F\left(x_{\epsilon} \mid t\right) d t
\end{aligned}
$$

provided we define $x(t)$ to be constant on $t \leqq 0$ and on $1 \leqq t$. Thus

$$
\begin{gathered}
G_{F \epsilon}[x]=\int_{0}^{1+\epsilon} F\left(x_{\epsilon} \mid t\right) d x_{\epsilon}(t), \\
G_{F \epsilon}[x]-G_{F}\left[x_{\epsilon}\right]=\int_{1}^{1+\epsilon} F\left(x_{\epsilon} \mid t\right) d x_{\epsilon}(t)=F\left(x_{\epsilon} \mid 1\right) \int_{1}^{1+\epsilon} d x_{\epsilon}(t) \\
=F\left(x_{\epsilon} \mid 1\right)\left[x_{\epsilon}(1+\epsilon)-x_{\epsilon}(1)\right] .
\end{gathered}
$$

Since $x_{\mathrm{e}} \rightarrow x$ and $F(x \mid 1)$ is continuous at $x=x_{0}$, we have

$$
\lim _{\epsilon \rightarrow 0+}\left\{G_{F_{\epsilon}}\left[x_{0}\right]-G_{F}\left[x_{0, \epsilon}\right]\right\}=0 .
$$

But by hypothesis, $G_{F}[x]$ is continuous at $x=x_{0}$, and hence

$$
\lim _{\epsilon \rightarrow 0+} G_{F}\left[x_{0, \epsilon}\right]=G_{F}\left[x_{0}\right] ;
$$

and (2.2), (2.3) with (1.10) and (2.1) imply (1.11). Thus the first part of the theorem is established, and the second part follows from the fact that

$$
G_{F}[x]=F(x \mid 1) x(1)-\int_{0}^{1} x(t) d F(x \mid t),
$$


since this implies that

$$
\begin{aligned}
\left|G_{F}[x]-G_{F}\left[x_{0}\right]\right| \leqq & \left|F(x \mid 1) x(1)-F\left(x_{0} \mid 1\right) x_{0}(1)\right| \\
& +||\left|x-x_{0}\right||| \operatorname{var}_{t \in I}\left[F\left(x_{0} \mid t\right)\right] \\
& +|||x||| \operatorname{var}_{t \in I}\left[F(x \mid t)-F\left(x_{0} \mid t\right)\right] .
\end{aligned}
$$

Thus Theorem 1 is established.

Proof of Theorem 2. By hypothesis it is clear that $F(x \mid 1)$ is continuous for each $x$ in $C$, and from the proof of Theorem 1 it follows that (2.2) holds. Thus, to establish (1.12) it only remains to show that

$$
\lim _{\epsilon \rightarrow 0+} G_{F}\left[x_{\epsilon}\right]=g(1, x(1))-\int_{0}^{1} g_{t}(t, x(t)) d t .
$$

We first note from (1.13) that $g(t, 0)=0$ and $g_{u}(t, u)=f(t, u)$ and that $g_{t}(t, u)$ $=\int_{0}^{u} f_{t}(t, v) d v$. We now have

$$
\begin{aligned}
G_{F}\left[x_{\epsilon}\right] & =\int_{0}^{1} F\left(x_{\epsilon} \mid t\right) d x_{\epsilon}(t)=\int_{0}^{1} g_{u}\left(t, x_{\epsilon}(t)\right) \frac{d x_{\epsilon}(t)}{d t} d t \\
& =\int_{0}^{1}\left\{\frac{d}{d t} g\left(t, x_{\epsilon}(t)\right)-g_{t}\left(t, x_{\epsilon}(t)\right)\right\} d t \\
& =g\left(1, x_{\epsilon}(1)\right)-\int_{0}^{1} g_{t}\left(t, x_{\epsilon}(t)\right) d t .
\end{aligned}
$$

Thus clearly (2.4) (and hence (1.12)) follows.

3. Statements of earlier results. Since we shall rely quite heavily on the statement of Theorem V of [2], we shall state it here in complete detail.

Theorem A. Let $\Gamma$ be any Wiener measurable subset of $C$, and assume that the transformation (1.1) takes $\Gamma$ into $T \Gamma$ in a 1-to-1 manner, and that $\Lambda$ is of smooth variation in a Hilbert neighborhood $H\left(x_{0}\right)$ of each point $x_{0}$ of $\Gamma$. Assume further that the following eight conditions hold in $H\left(x_{0}\right)$ for each $x_{0} \in \Gamma$ :

(3.1.1) $\Lambda(x \mid t) \in C$ and $\Lambda_{t}(x \mid t)$ exists and is of bounded variation in $t$ on $I$ for each $x \in H\left(x_{0}\right)$.

(3.1.2) $K(x \mid 0, s)=0$ for $(x, s) \in H\left(x_{0}\right) \otimes I$.

(3.1.3) $\left\|K\left(x_{1} \mid t_{1}, \cdot\right)-K\left(x_{2} \mid t_{2}, \cdot\right)\right\| \rightarrow 0$ uniformly for $t_{1}, t_{2} \in I$ and $x_{1}, x_{2} \in H\left(x_{0}\right)$ as $\left|t_{1}-t_{2}\right| \rightarrow 0$ and $\left\|x_{1}-x_{2}\right\| \rightarrow 0$.

(3.1.4) $\|K(x \mid t, \cdot)\|$ is bounded in $H\left(x_{0}\right) \otimes I$.

(3.1.5) $D\left(x_{0}\right) \neq 0$.

(3.1.6) $K_{t}(x \mid t, s)$ exists and is continuous (uniform topology for $x$ ) in $H\left(x_{0}\right) \otimes I^{2}$.

(3.1.7) $\sup _{t, s \in I}\left|K_{t}(x \mid t, s)\right|$ (and hence also $\sup _{t, s \in I}|K(x \mid t, s)|$ ) is bounded in every subset of $H\left(x_{0}\right)$ which is bounded in the uniform topology. 
(3.1.8) $\sup _{s \in I} \operatorname{var}_{t \in I}\left[K_{t}(x \mid t, s)\right]$ is bounded in every subset of $H\left(x_{0}\right)$ which is bounded in the uniform topology.

Then it follows that $T \Gamma$ is Wiener measurable, and furthermore that if $F(y)$ is a Wiener measurable functional that makes either side of the following exist, both sides exist and they are equal:

$$
\begin{aligned}
\int_{T \Gamma}^{w} F(y) d_{w} y=\int_{\Gamma}^{w} F(x+\Lambda(x \mid \cdot)) \exp ( & -2 \int_{0}^{1} \Lambda_{t}(x \mid t) d x(t) \\
& \left.-\int_{0}^{1}\left[\Lambda_{t}(x \mid t)\right]^{2} d t\right)|D(x)| d_{w} x .
\end{aligned}
$$

In addition to this theorem we shall need the following lemma which is almost identical with Lemma 1 of [2] in statement and proof.

LEMma 1. Let $\Lambda(x \mid t)$ be a functional of semi-smooth variation defined on $S \otimes I$, where $S$ is an open convex subset of $C$, and let $K(x \mid t, s)$ be the kernel of its variation. Then if $x_{0} \in S$, we have

$$
\Lambda(x \mid t)=\Lambda\left(x_{0} \mid t\right)+\int_{0}^{1} d v \int_{0}^{1} K\left[x_{0}+\left(x-x_{0}\right) v \mid t, s\right]\left[x(s)-x_{0}(s)\right] d s
$$

for all $(x, t) \in S \otimes I$. Moreover the kernel $K$ is completely defined in $S \otimes I^{2}$ by the functional $\Lambda$.

Finally, we state Lemma 2 as a trivial extension of Corollary 3 of Lemma 2 of $[1]$.

Lemma 2. Let $z_{0}$ be a limit point of a set $Z$ in a metric space $\subseteq$, let $K(z \mid t, s)$ be Borel measurable in $(t, s)$ for each $z \in Z$ on $0<t, s<1$, let $K$ be bounded on $Z \otimes I^{2}$, and let $\lim _{z \rightarrow z_{0}} K(z \mid t, s)=K(t, s)$ on $0<t, s<1$. Then $\lim _{z \rightarrow z_{0}} D(z)=D$, where $D(z)$ and $D$ are respectively the Fredholm determinants associated with $K(z \mid t, s)$ and $K(t, s)$, with parameter $\lambda=-1$.

4. Statements of the restricted theorems. Before proving Theorems 3 and 4 we shall have to prove the following two weaker theorems.

THEOREM 3a. The conclusion of Theorem 3 holds if we add to the hypothesis the assumption

$$
\operatorname{var}_{t \in I}[K(x \mid t, s)] \text { is finite and is bounded on } S_{0} \otimes I .
$$

THEOREM 4a. The conclusion of Theorem 4 holds if we add to the hypothesis that condition (4.1) is to hold in $S_{0}\left(x_{0}\right)$ for each $x_{0}$ in $\Gamma$.

5. Behavior of the smoothed $\Lambda$ and its kernel. We shall next prove some preliminary lemmas that will be used in establishing Theorem $3 \mathrm{a}$, which is 
the crux of the paper. In this section we shall prove Lemmas 3,4 , and 5 , which give the behavior of a suitably smoothed modification of $\Lambda$.

Notation. Let

$$
\Lambda^{\epsilon}(x \mid t) \equiv \Lambda_{\epsilon}(x \mid t)-\Lambda_{\epsilon}(x \mid 0)
$$

where $\Lambda_{\epsilon}$ has the meaning given by the $\epsilon$-subscript notation defined in (1.9). Let $T^{\epsilon}$ denote the transformation

$$
T^{\epsilon}: \quad y(t)=x(t)+\Lambda^{\epsilon}(x \mid t) ;
$$

and let $\Lambda^{0}, K^{0}, T^{0}$, and $D^{0}$ denote $\Lambda, K, T$, and $D$ respectively.

Lemma 3. Let $\Lambda(x \mid t)$ satisfy the hypotheses of Theorem 3. Then there is a concentric subsphere $S_{1}$ of $S_{0}$ and a positive number $\epsilon_{1}$ such that if $x_{0}^{\prime} \in S_{1}$ and $0<\epsilon<\epsilon_{1}, \Lambda^{*}(x \mid t)$ is of smooth variation in some Hilbert neighborhood $H_{\epsilon}\left(x_{0}^{\prime}\right)$ of $x_{0}^{\prime}$. Moreover if $(x, t, s) \in H_{\epsilon}\left(x_{0}^{\prime}\right) \otimes I^{2}$, the variation kernel $K^{\epsilon}$ of $\Lambda^{e}$ is given by

$$
\begin{aligned}
& K^{\epsilon}(x \mid t, s) \\
& \quad=\frac{1}{\epsilon^{2}} \int_{s}^{\min (s+\epsilon, 1)}\left\{\int_{t}^{t+\epsilon} K\left(x_{\epsilon} \mid \tau, u\right) d \tau-\int_{0}^{\epsilon} K\left(x_{\epsilon} \mid \tau, u\right) d \tau\right\} d u .
\end{aligned}
$$

Proof. As a preliminary step we notice the following result which will be of use in other lemmas as well as this one. Let $r_{0}$ be the radius of $S_{0}$ and let $S_{1}$ be the uniform sphere of center $x_{0}$ and radius $r_{0} / 3$. Let $x_{0, \mathrm{e}}(t)$ be the lower $\epsilon$-smoothing of $x_{0}(t)$; choose $\epsilon_{1}>0$ such that $\left\|\left|x_{0, \epsilon}-x_{0} \|\right|<r_{0} / 3\right.$ if $0<\epsilon<\epsilon_{1}$; and let $H_{\epsilon}\left(x_{0}^{\prime}\right)$ be the Hilbert sphere of radius $\left(r_{0} / 3\right) \epsilon^{1 / 2}$ about $x_{0}^{\prime}$. Then if $0<\epsilon<\epsilon_{1}$ and $x_{0}^{\prime} \in S_{1}$, we have

$$
x \in H_{\epsilon}\left(x_{0}^{\prime}\right) \rightarrow x_{\epsilon} \in S_{0} .
$$

For

$$
\left\||| x_{\epsilon}-x_{0}|\|\leqq\||\left|x_{\epsilon}-x_{0, \epsilon}^{\prime}\right|||+||\left|x_{0, \epsilon}^{\prime}-x_{0, \epsilon}\|||+\|\right|\left|x_{0, \epsilon}-x_{0}\right|\right\|,
$$

and, using the Schwarz inequality for the first term, we see that each term on the right is less than $r_{0} / 3$ under the given circumstances. It is to be noted that the radius of $S_{1}$ (namely $r_{0} / 3$ ) is independent of $\epsilon$; and it should also be noted that if $x \in H_{\epsilon}\left(x_{0}^{\prime}\right)$ we have that $\Lambda\left(x_{\epsilon} \mid t\right)$ is defined and $\Lambda(y \mid t)$ is of semi-smooth variation in a set containing $y=x_{\boldsymbol{c}}$.

We next show that $\Lambda^{\epsilon}(x \mid t)$ is of smooth variation in $H_{\epsilon}\left(x_{0}^{\prime}\right)$ and compute its kernel $K^{\epsilon}(x \mid t, s)$. Using the definition of $\Lambda^{\epsilon}$ in (5.1) and (1.2), (1.3), (1.8), (1.9) and the fact that $K(x \mid s, u)=K(x \mid s, 1)$ on $1 \leqq s<\infty$ and $y(t)=0$ if $t<0$, we have for $(x, y, t) \in H_{\epsilon}\left(x_{0}^{\prime}\right) \otimes C \otimes I$

$$
\begin{gathered}
\delta \Lambda^{\epsilon}(x|t| y)=\delta \Lambda_{\epsilon}(x|t| y)+\delta \Lambda_{\epsilon}(x|0| y), \\
\left.\delta \Lambda_{\epsilon}(x|t| y)=\frac{\partial}{\partial v}\left\{\frac{1}{\epsilon} \int_{t}^{t+\epsilon} \Lambda\left(x_{\epsilon}-v y_{\epsilon} \mid \tau\right) d \tau\right\}\right]_{v=0}=\frac{1}{\epsilon} \int_{t}^{t+\epsilon} \delta \Lambda\left(x_{\epsilon}|\tau| y_{\epsilon}\right) d \tau .
\end{gathered}
$$




$$
\begin{aligned}
& =\frac{1}{\epsilon} \int_{t}^{t+\epsilon} \int_{0}^{1} K\left(x_{\epsilon} \mid \tau, u\right) y_{\epsilon}(u) d u d \tau \\
& =\frac{1}{\epsilon^{2}} \int_{0}^{1}\left\{\int_{u-\epsilon}^{u} y(s) d s \int_{t}^{t+\epsilon} K\left(x_{\epsilon} \mid \tau, u\right) d \tau\right\} d u \\
& =\frac{1}{\epsilon^{2}} \int_{0}^{1} y(s) d s \int_{s}^{\min (s+\epsilon, 1)} d u \int_{t}^{t+\epsilon} K\left(x_{\epsilon} \mid \tau, u\right) d \tau .
\end{aligned}
$$

Thus $\delta \Lambda^{\epsilon}$ is of the form (1.3) with kernel $K^{\epsilon}$ given by (5.2), and it follows from the hypotheses on $K$ that $K^{e}$ is continuous in $(x, t, s)$ throughout $H_{\epsilon}\left(x_{0}^{\prime}\right) \otimes I^{2}$ whenever $x_{0}^{\prime} \in S_{1}$ and $0<\epsilon<\epsilon_{1}$. This completes the proof of the lemma.

In the following lemmas (4-7), the notation introduced in connection with Lemma 3 will be maintained, and it will be assumed in all of them that $\Lambda$ satisfies the hypotheses of Theorem 3 .

Lemмa 4. If $B$ denotes an upper bound for $K(x \mid t, s)$ in $S_{0} \otimes I^{2}$, we have

$$
\begin{aligned}
& \left|K^{\epsilon}(x \mid t, s)\right| \leqq 2 B \text { for }(x, t, s, \epsilon) \in S_{1} \otimes I^{2} \otimes\left[0, \epsilon_{1}\right) \text { and } \\
& \qquad \text { for } x \in H_{\epsilon}\left(x_{0}^{\prime}\right) \text { with }\left(x_{0}^{\prime}, t, s, \epsilon\right) \in S_{1} \otimes I^{2} \otimes\left(0, \epsilon_{1}\right) .
\end{aligned}
$$

If $x_{0}^{\prime} \in S_{1}$ and $0<\epsilon<\epsilon_{1}$, then $\Lambda^{\epsilon}(x \mid t)$ and $K^{\epsilon}(x \mid t, s)$ satisfy conditions (3.1.1)(3.1.4) and (3.1.6), (3.1.7) (with $x_{0}^{\prime}$ replacing $x_{0}$ and $H_{\epsilon}$ replacing $H$ ). Moreover if $K$ satisfies condition (4:1), $K^{\epsilon}$ satisfies condition (3.1.8).

Proof. The inequality (5.4) follows trivially from (5.2), (5.3). Each of the conditions can be verified in a straightforward manner, and we shall therefore carry through the verification only for the most difficult one, namely (3.1.3). We observe

$$
\begin{aligned}
\left\|K^{\epsilon}\left(x_{1} \mid t_{1}, \cdot\right)-K^{\epsilon}\left(x_{2} \mid t_{2}, \cdot\right)\right\| \leqq & || K^{\epsilon}\left(x_{1} \mid t_{1}, \cdot\right)-K^{\epsilon}\left(x_{2} \mid t_{2}, \cdot\right)|| \\
= & \frac{1}{\epsilon^{2}} \sup _{s \in I} \mid \int_{s}^{\min (s+\epsilon, 1)}\left\{\int_{t_{1}}^{t_{1}+\epsilon} K\left(x_{1, \epsilon} \mid \tau, u\right) d \tau\right. \\
& \left.-\int_{t_{2}}^{t_{2}+\epsilon} K\left(x_{2, \epsilon} \mid \tau, u\right) d \tau-\int_{0}^{\epsilon}\left[K\left(x_{1, \epsilon} \mid \tau, u\right)-K\left(x_{2, \epsilon} \mid \tau, u\right)\right] d \tau\right\} d u \mid \\
(5.5) \leqq & \frac{1}{\epsilon^{2}} \int_{0}^{1}\left|\int_{t_{1}}^{t_{2}} K\left(x_{1, \epsilon} \mid \tau, u\right) d \tau-\int_{t_{1}+\epsilon}^{t_{2}+\epsilon} K\left(x_{1, \epsilon} \mid \tau, u\right) d \tau\right| d u . \\
& +\frac{1}{\epsilon^{2}} \int_{0}^{1} d u \int_{t_{2}}^{t_{2}+\epsilon}\left|K\left(x_{1, \epsilon} \mid \tau, u\right)-K\left(x_{2, \epsilon} \mid \tau, u\right)\right| d \tau \\
& +\frac{1}{\epsilon^{2}} \int_{0}^{1} d u \int_{0}^{\epsilon}\left|K\left(x_{1, \epsilon} \mid \tau, u\right)-K\left(x_{2, \epsilon} \mid \tau, u\right)\right| d \tau .
\end{aligned}
$$

Now the first term of the last member does not exceed $2 \epsilon^{-2}\left|t_{2}-t_{1}\right| B$, and by 
the Schwarz inequality each of the last two terms of the last member of (5.5) does not exceed

$$
\frac{1}{\epsilon^{2}} \int_{0}^{1}\left\|K\left(x_{1, \epsilon} \mid \tau, \cdot\right)-K\left(x_{2, \epsilon} \mid \tau, \cdot\right)\right\| d \tau .
$$

Hence by (1.14.3) and (1.14.4) and the principle of bounded convergence it follows that the last two terms of (5.5) approach zero uniformly for $x_{1}, x_{2}$ $\in H_{\epsilon}\left(x_{0}^{\prime}\right)$ as $\left\|x_{1}-x_{2}\right\| \rightarrow 0$. Thus (3.1.3) holds in $H_{\epsilon}\left(x_{0}^{\prime}\right)$. This concludes the proof of Lemma 4.

LEMMA 5. We have

$$
\lim _{x \rightarrow x_{0}} K(x \mid t, s)=K\left(x_{0} \mid t, s\right) \text { uniformly for }(t, s) \in I^{2}
$$

$$
\lim _{\epsilon \rightarrow 0^{+}, x \rightarrow x_{0}}\left[K^{\epsilon}(x \mid t, s)-K^{\epsilon}\left(x_{0} \mid t, s\right)\right]=0 \text { uniformly for }(t, s) \in I^{2}
$$

$$
\lim _{c \rightarrow 0^{+}, \nu \rightarrow x} K^{e}(y \mid t, s)=K(x \mid t, s) \text { if }(x, t, s) \in S_{1} \otimes\{0<t, s<1\} ;
$$

$$
\lim _{\epsilon \rightarrow 0^{+}, y \rightarrow x} \Lambda^{\epsilon}(y \mid t)=\Lambda(x \mid t) \text { if }(x, t) \in S_{1} \otimes I \text { uniformly in } t \text { for each } x
$$

$$
\lim _{\substack{\rightarrow 0^{+}, y \rightarrow x\\}} D^{\epsilon}(y)=D(x) \text { if } x \in S_{1} .
$$

Here $D^{\epsilon}$ denotes the Fredholm determinant associated with $K^{\epsilon}$.

Proof. Since $\Lambda$ is of semi-smooth variation, $K^{1}(x \mid t, s)$ is continuous in $(x, t, s)$ in $S_{0} \otimes\{0 \leqq t \leqq s \leqq 1\}$ and hence for each $x$ in $S_{0}$ it is continuous in $x$ uniformly with respect to $(t, s)$ in $\{0 \leqq t \leqq s \leqq 1\}$. The same is true for $K^{2}(x \mid t, s)$ in $\{0 \leqq s \leqq t \leqq 1\}$ and for $K^{1}+K^{2}$ on the diagonal $\{0 \leqq s=t \leqq 1\}$. Hence for each $x$ in $S_{0}, K(x \mid t, s)$ is continuous in $x$ uniformly with respect to $(t, s)$ in $I^{2}$, and in particular, (5.6.1) is established. Moreover, as $x \rightarrow x_{0}$ and $\epsilon \rightarrow 0^{+}$, we have $x_{0, \epsilon} \rightarrow x_{0}$ and $x_{\epsilon} \rightarrow x_{0}$ and $K\left(x_{0, \epsilon} \mid t, s\right)$ and $K\left(x_{\epsilon} \mid t, s\right)$ both approach $K\left(x_{0} \mid t, s\right)$ uniformly in $t, s$. Thus $K\left(x_{\epsilon} \mid t, s\right)-K\left(x_{0, \epsilon} \mid t, s\right) \rightarrow 0$ uniformly in $(t, s)$ in $I^{2}$, and (5.6.2) follows from (5.2) and (5.6.1).

Again, if $0<t, s<1$ and $x, y \in S_{1}$ and $0<\epsilon<\min \left(\epsilon_{1}, 1-s\right)$, we have from

$$
\begin{aligned}
K^{\epsilon}(y \mid t, s)-K(x \mid t, s)= & \frac{1}{\epsilon^{2}} \int_{s}^{8+\epsilon} \int_{t}^{t+\epsilon}\left[K\left(y_{\epsilon} \mid \tau, u\right)-K(x \mid t, s)\right] d \tau d u \\
& -\frac{1}{\epsilon^{2}} \int_{s}^{s+\epsilon} \int_{0}^{\epsilon} K\left(y_{\epsilon} \mid \tau, u\right) d \tau d u
\end{aligned}
$$

Since $K$ is continuous in $(x, t, s)$ when $t \neq s$ and since $K(x \mid 0, s)=0$, it follows that the two averages in the right member of (5.7) approach zero as $\epsilon \rightarrow 0^{+}$ and $y \rightarrow x$ (in the uniform topology), provided $t \neq s$. On the other hand, if 
$t=s$, we have $K=K^{1} / 2+K^{2} / 2$ and hence for $0<\epsilon<\min \left(\epsilon_{1}, t, 1-t\right), 0<t<1$, and $x \in S_{1}$ we have

$$
\begin{aligned}
K^{\epsilon}(y \mid t, t)-K(x \mid t, t)= & \frac{1}{\epsilon^{2}} \int_{t}^{t+\epsilon} \int_{t}^{u}\left[K^{1}\left(y_{\epsilon} \mid \tau, u\right)-K^{1}(x \mid t, t)\right] d \tau d u \\
& +\frac{1}{\epsilon^{2}} \int_{t}^{t+\epsilon} \int_{u}^{t+\epsilon}\left[K^{2}\left(y_{\epsilon} \mid \tau, u\right)-K^{2}(x \mid t, t)\right] d \tau d u \\
& -\frac{1}{\epsilon^{2}} \int_{t}^{t+\epsilon} \int_{0}^{\epsilon} K^{1}\left(y_{\epsilon} \mid \tau, u\right) d \tau d u .
\end{aligned}
$$

As before, the averages on the right approach zero as $\epsilon \rightarrow 0^{+}$and $y \rightarrow x$, and hence, whether $t=s$ or not, we have (5.6.3). Moreover it follows from (5.6.3), (5.4), and Lemma 2 that (5.6.5) holds.

Finally it follows from (1.14.1) that $\Lambda_{t}(x \mid t)$ is continuous in $(x, t)$ on $S_{0} \otimes I$ uniformly in $t$ for each $x$, and by integration on $t$ that the same is true for $\Lambda$. Thus for each $x \in S_{1}, \Lambda\left(y_{\epsilon} \mid s\right) \rightarrow \Lambda(x \mid t)$ uniformly in $t$ as $y \rightarrow x, s \rightarrow t$, and $\epsilon \rightarrow 0^{+}$; and hence $\Lambda_{\epsilon}(y \mid t) \rightarrow \Lambda(x \mid t)$ uniformly in $t$ and (5.6.4) holds. Thus the lemma is proved.

6. Behavior of smoothed transformations. We shall now study the smoothed transformation

$$
T^{\epsilon}: \quad y(t)=x(t)+\Lambda^{\epsilon}(x \mid t) .
$$

In the following two lemmas, the notation used in Lemma 3 and the hypotheses of Theorem 3 will continue to be understood.

LEMMA 6. There is a uniform concentric subsphere $S_{2}$ of $S_{1}$ and a positive $\epsilon_{2} \leqq \epsilon_{1}$ such that $T^{\epsilon}$ takes $S_{2}$ into $T^{\epsilon} S_{2}$ in a 1-to-1 manner for each $\epsilon$ on $0 \leqq \epsilon \leqq \epsilon_{2}$. The radius of $S_{2}$ is independent of $\epsilon$.

Proof. Consider the transformation

$$
T_{1}^{\epsilon}: \quad y(t)=x(t)+\stackrel{\epsilon}{\epsilon}(x \mid t),
$$

where

$$
\Lambda_{1}^{\epsilon}(x \mid t)=\Lambda^{\epsilon}\left(x_{0} \mid t\right)+\int_{0}^{1} K^{\epsilon}\left(x_{0} \mid t, s\right)\left[x(s)-x_{0}(s)\right] d s .
$$

Now by (5.6.5) and (1.14.5) we can choose a positive $\epsilon_{2}^{\prime} \leqq \epsilon_{1}$, a concentric subsphere $S_{2}^{\prime}$ of $S_{1}$, and a positive number $\gamma$ such that

$$
\left|D^{e}(x)\right|>\gamma>0 \quad \text { if } x \in S_{2}^{\prime} \text { and } 0 \leqq \epsilon \leqq \epsilon_{2}^{\prime} ;
$$

so it follows from Fredholm theory that the linear transformation $T_{1}^{e}$ has a single-valued inverse $\left(T_{1}^{\epsilon}\right)^{-1}$ over $C$ given by 


$$
\begin{aligned}
\left(T_{1}^{e}\right)^{-1}: z(t)= & y(t)-\Lambda^{\epsilon}\left(x_{0} \mid t\right) \\
& +\int_{0}^{1} k^{\epsilon}(t, s)\left[y(s)-x_{0}(s)-\Lambda^{\epsilon}\left(x_{0} \mid t\right)\right] d s,
\end{aligned}
$$

where $k^{e}(t, s)$ is the Volterra reciprocal kernel of $K^{\bullet}\left(x_{0} \mid t, s\right)$ and hence satis. fies for $0 \leqq \epsilon \leqq \epsilon_{2}^{\prime}$

$$
k^{\epsilon}(t, s)+K^{\epsilon}\left(x_{0} \mid t, s\right)=-\int_{0}^{1} k^{\epsilon}(t, u) K^{\epsilon}\left(x_{0} \mid u, s\right) d u .
$$

It is easy to show by integral equation theory that $k^{e}(t, s)$ is bounded for $(t, s, \epsilon) \in I^{2} \otimes\left[0, \epsilon_{2}^{\prime}\right]$ since we have already shown in (5.4) and (6.3) that $K^{\epsilon}\left(x_{0} \mid t, s\right)$ and $\left[D^{\epsilon}\left(x_{0}\right)\right]^{-1}$ are. Let

$$
\left|k^{\epsilon}(t, s)\right|<B_{1} \quad \text { for }(t, s, \epsilon) \in I^{2} \otimes\left[0, \epsilon_{2}^{\prime}\right] .
$$

Now let

$$
T_{2}^{\epsilon}=\left(T_{1}^{\epsilon}\right)^{-1} T^{\epsilon}
$$

so that

$$
T_{2}^{\epsilon}: \quad z(t)=x(t)+\Lambda_{2}^{\epsilon}(x \mid t)
$$

where

$$
\begin{aligned}
\Lambda_{2}^{e}(x \mid t)= & \Lambda^{\epsilon}(x \mid t)-\Lambda^{\epsilon}\left(x_{0} \mid t\right) \\
& +\int_{0}^{1} k^{\epsilon}(t, s)\left[x(s)+\Lambda^{c}(x \mid s)-x_{0}(s)-\Lambda^{\epsilon}\left(x_{0} \mid s\right)\right] d s .
\end{aligned}
$$

We shall now obtain a Lipschitz condition for $\Lambda_{2}^{\mathbf{e}}$.

To estimate the increment of $\Lambda^{\boldsymbol{e}}$ as $x$ changes, we apply Lemma 1 to $\Lambda^{\bullet}(x \mid t)$ and obtain for $x, x^{\prime} \in S_{2}^{\prime}$

$$
\begin{aligned}
I_{1}(t) & \equiv \Lambda^{\epsilon}(x \mid t)-\Lambda^{\epsilon}\left(x^{\prime} \mid t\right) \\
& =\int_{0}^{1} d v \int_{0}^{1} K^{\epsilon}\left[x^{\prime}+\left(x-x^{\prime}\right) v \mid t, s\right]\left[x(s)-x^{\prime}(s)\right] d s ;
\end{aligned}
$$

and from (6.8) it follows that

$$
\Lambda_{2}^{e}(x \mid t)-\Lambda_{2}^{e}\left(x^{\prime} \mid t\right)=I_{1}(t)+I_{2}(t)
$$

where

$$
I_{2}(t)=\int_{0}^{1} k^{e}(t, s)\left[x(s)-x^{\prime}(s)+I_{1}(s)\right] d s .
$$


Substituting (6.9) in (6.11) we have

$$
\begin{aligned}
I_{2}(t)= & \int_{0}^{1} k^{\epsilon}(t, s)\left[x(s)-x^{\prime}(s)\right] d s \\
& +\int_{0}^{1} k^{\epsilon}(t, s) d s \int_{0}^{1} K^{\epsilon}\left(x_{0} \mid s, u\right)\left[x(u)-x^{\prime}(u)\right] d u \\
& +\int_{0}^{1} k^{\epsilon}(t, s) d s \int_{0}^{1} d v \int_{0}^{1}\left[K^{\epsilon}\left(x^{\prime}+\left(x-x^{\prime}\right) v \mid s, u\right)\right. \\
& \left.-K^{e}\left(x_{0} \mid s, u\right)\right]\left[x(u)-x^{\prime}(u)\right] d u .
\end{aligned}
$$

Simplifying the second term of the right member of (6.12) by means of (6.5), cancelling the first term with one of the resulting terms, and substituting in (6.10), we obtain (using (6.9)),

$$
\begin{aligned}
\Lambda_{2}^{\prime}(x \mid t)- & \Lambda_{2}^{\epsilon}\left(x^{\prime} \mid t\right) \\
= & \int_{0}^{1} d v \int_{0}^{1}\left[K^{\epsilon}\left(x^{\prime}+\left(x-x^{\prime}\right) v \mid t, s\right)-K^{\epsilon}\left(x_{0} \mid t, s\right)\right]\left[x(s)-x^{\prime}(s)\right] d s \\
& +\int_{0}^{1} k^{\epsilon}(t, s) d s \int_{0}^{1} d v \int_{0}^{1}\left[K^{\epsilon}\left(x^{\prime}+\left(x-x^{\prime}\right) v \mid s, u\right)\right. \\
& \left.-K^{\epsilon}\left(x_{0} \mid s, u\right)\right]\left[x(u)-x^{\prime}(u)\right] d u,
\end{aligned}
$$

so that from (6.6) it follows that

$$
\left\||| \Lambda_{2}^{e}(x \mid \cdot)-\Lambda_{2}^{e}\left(x^{\prime} \mid \cdot\right)\right\|\left|\leqq\left(1+B_{1}\right)\left\||| x-x^{\prime}\right\|\right| G\left(x, x^{\prime}\right)
$$

where, for $x, x^{\prime} \in S_{2}^{\prime}$,

$$
\text { (6.14) } G\left(x, x^{\prime}\right)=\sup _{v, t \in I} \int_{0}^{1}\left|K^{\epsilon}\left(x^{\prime}+\left(x-x^{\prime}\right) v \mid t, s\right)-K^{\epsilon}\left(x_{0} \mid t, s\right)\right| d s \text {. }
$$

We next seek to estimate $G\left(x, x^{\prime}\right)$ in the neighborhood of $x_{0}$. We note that for $v \in I$,

$$
\begin{aligned}
\left\|\left[x^{\prime}+\left(x-x^{\prime}\right) v\right]-x_{0}\right\| & \leqq v\|\| x-x_{0}\|\|+(1-v)\|\| x^{\prime}-x_{0}\|\| \\
& \leqq \max \left[\left\||| x-x_{0}\right\|\|,\||| x^{\prime}-x_{0}\|\|\right]
\end{aligned}
$$

so that we have from (5.6.2) and (6.14) that $G\left(x, x^{\prime}\right) \rightarrow 0$ as $x \rightarrow x_{0}, x^{\prime} \rightarrow x_{0}$, and $\epsilon \rightarrow 0^{+}$. We can therefore choose a concentric subsphere $S_{2}^{\prime \prime}$ of $S_{2}^{\prime}$ and a positive number $\epsilon_{2} \leqq \epsilon_{2}^{\prime}$ such that

$$
G\left(x, x^{\prime}\right) \leqq \frac{1}{6\left(1+B_{1}\right)}
$$
when $x, x^{\prime} \in S_{2}^{\prime \prime}$ and $0 \leqq \epsilon \leqq \epsilon_{2}$. 
Thus we have for $0 \leqq \epsilon \leqq \epsilon_{2}$,

$$
\left\||| \Lambda_{2}^{e}(x \mid \cdot)-\Lambda_{2}^{\epsilon}\left(x^{\prime} \mid \cdot\right)\right\||| \leqq \frac{1}{6}\left\||| x-x^{\prime} \mid\right\|, \quad x, x^{\prime} \in S_{2}^{\prime \prime} .
$$

The radius $r_{2}^{\prime \prime}$ of $S_{2}^{\prime \prime}$ is independent of $\epsilon$.

We have obtained a Lipschitz condition for $\Lambda_{2}^{e}$, and we shall now show that a transformation can be defined which agrees with $\Lambda_{2}$ in the neighborhood of $x_{0}$, is defined over the whole of $C$, and satisfies a Lipschitz condition with multiplier less than unity. This will enable us to apply Lemma 2 of [2] and show that the new transformation takes $C$ into $C$ in a 1-to-1 way.

Let the transformation $T_{3}^{\epsilon}$ be defined over the whole of $C$ for $0 \leqq \epsilon \leqq \epsilon_{2}$ as follows:

$$
T_{3}^{\epsilon}: \quad y(t)=x(t)+\Lambda_{3}^{\epsilon}(x \mid t)
$$

where

$$
\Lambda_{3}^{\epsilon}(x \mid t)=\Lambda_{2}^{\epsilon}(x \mid t) \psi\left(\theta||\left|x-x_{0}\right|||\right)
$$

and

$$
\theta=2 / r_{2}^{\prime \prime}
$$

and $\Lambda_{2}^{\epsilon}$ is defined in any arbitrary manner where it is now undefined $\left(^{3}\right)$, and

$$
\psi(u)=\left\{\begin{array}{cll}
1 & \text { if } & u \leqq 1 \\
2-u & \text { if } & 1 \leqq u \leqq 2 \\
0 & \text { if } & 2 \leqq u
\end{array}\right.
$$

We note that the function $\psi$ satisfies the Lipschitz condition

$$
\left|\psi\left(u_{1}\right)-\psi\left(u_{2}\right)\right| \leqq\left|u_{1}-u_{2}\right|
$$

for non-negative $u_{1}, u_{2}$. We shall show that for all sufficiently small $\epsilon, \Lambda_{3}^{\dot{\varepsilon}}$ satisfies the Lipschitz condition

$$
\left\||| \Lambda_{3}^{c}(x \mid \cdot)-\Lambda_{3}^{c}\left(x^{\prime} \mid \cdot\right)\right\|\left\|\leqq \frac{1}{2}\right\||| x-x^{\prime} \|, \quad x, x^{\prime} \in C .
$$

To show this we assume without loss of generality (because of the symmetry of (6.21)) that

$$
\left\||| x-x_{0}||\left|\geqq\left\||| x^{\prime}-x_{0} \mid\right\|\right. \text {. }\right.
$$

From the definition of $\Lambda_{3}^{\epsilon}(x \mid t)$ we have that

$$
||\left|\Lambda_{3}^{e}(x \mid \cdot)-\Lambda_{3}^{e}\left(x^{\prime} \mid \cdot\right)\right| \| \leqq Q_{1}+Q_{2}
$$

where

(3) The values of $\Lambda_{2}^{\epsilon}$ outside $S_{2}^{\prime \prime}$ are of no significance because we have chosen $\theta$ so that $\psi\left(\theta||\left|x-x_{0}\right| \|\right)$ is zero there. 


$$
Q_{1}=\left\||| \Lambda_{2}^{e}(x \mid \cdot)-\Lambda_{2}^{e}\left(x^{\prime} \mid \cdot\right)\right\| \| \psi\left(\theta||\left|x-x_{0} \|\right| \mid\right)
$$

and

$$
\text { (6.25) } Q_{2}=\left\||| \Lambda_{2}^{e}\left(x^{\prime} \mid \cdot\right)\right\| \|\left\{\mid \psi\left(\theta||\left|x-x_{0}\right|||\right)-\psi\left(\theta||\left|x^{\prime}-x_{0}\right|||\right)\right. \text {. }
$$

From (6.24), (6.15), (6.18), (6.19) we have for all $x, x^{\prime} \in C$ satisfying (6.22) and for $0 \leqq \epsilon \leqq \epsilon_{2}$,

$$
Q_{1} \leqq \frac{1}{6} \||| x-x^{\prime}|| \mid
$$

(as one sees by taking the cases $x \in S_{2}^{\prime \prime}$ and $x \notin S_{2}^{\prime \prime}$ separately). Next we note from (6.8) that $\Lambda_{2}^{\epsilon}\left(x_{0} \mid t\right) \equiv 0$ and from (6.25), (6.15), (6.20) we have for all $x \in C$ and $x^{\prime} \in S_{2}^{\prime \prime}$ and for $0 \leqq \epsilon \leqq \epsilon_{2}$,

$$
Q_{2} \leqq \frac{1}{6}||\left|x^{\prime}-x_{0}\right||| \cdot \theta||\left|x-x^{\prime}\right| \|,
$$

so that from (6.18), (6.25), (6.19) we have for all $x, x^{\prime} \in C$ satisfying (6.22) and for $0 \leqq \epsilon \leqq \epsilon_{2}$

$$
Q_{2} \leqq \frac{1}{3}||\left|x-x^{\prime} \|\right| \mid
$$

(as one sees by taking the cases $x^{\prime} \in S_{2}^{\prime \prime}$ and $x^{\prime} \notin S_{2}^{\prime \prime}$ separately). But (6.23), (6.26), and (6.27) imply that (6.21) holds whenever (6.22) holds, and by symmetry it follows that (6.21) holds for all $x, x^{\prime} \in C$, provided $0 \leqq \epsilon \leqq \epsilon_{2}$.

Now the Lipschitz condition (6.21) allows us to conclude from Picard's theorem (see Lemma 2 of [2]), that $T_{3}^{e}$ takes $C$ into $C$ in a 1-to-1 manner. Moreover, from (6.17) it follows that $\Lambda_{3}^{e}(x \mid t)=\Lambda_{2}^{e}(x \mid t)$ whenever $\psi\left(\theta||\left|x-x_{0}\right|||\right)$ $=1$, so that by (6.16), (6.18), (6.19), we have

$$
T_{2}^{\epsilon}(x)=T_{3}^{\epsilon}(x)
$$

if $0 \leqq \epsilon \leqq \epsilon_{2}$ and $x \in S_{2}$, where $S_{2}$ is the open sphere of radius $r_{2}=r_{2}^{\prime} / 2$ about $x_{0}$. Thus

$$
T_{2}^{\epsilon} S_{2}=T_{3}^{\epsilon} S_{2}, \quad 0 \leqq \epsilon \leqq \epsilon_{2},
$$

and $T_{2}^{\epsilon}$ takes $S_{2}$ into $T_{2}^{\epsilon} S_{2}$ in a 1-to-1 manner, so that

$$
\left(T_{2}^{\epsilon}\right)^{-1} y=\left(T_{3}^{\epsilon}\right)^{-1} y \quad \text { if } y \in T_{2}^{\epsilon} S_{2}, 0 \leqq \epsilon \leqq \epsilon_{2},
$$

where the left member of (6.30) is understood to be that value of the inverse which lies in $S_{2}$, and there is obviously only one such value. Moreover, since $T_{1}^{*}$ takes $C$ into $C$ in a 1-to-1 manner, it follows that $T_{1}^{*}$ takes $T_{2}^{e} S_{2}$ into $T_{1}^{\epsilon} T_{2}^{\epsilon} S_{2}$ in a 1-to-1 manner; or since $T_{1}^{\epsilon} T_{2}^{\epsilon}=T^{\bullet}$ by (6.7), we have that $T^{\bullet}$ takes $S_{2}$ into $T^{*} S_{2}$ in a 1-to-1 manner. Thus the lemma is established.

Lemma 7. For $0 \leqq \epsilon \leqq \epsilon_{2}$ the sets $T^{\epsilon} S_{2}$ are open. Moreover there exists a uniform sphere $U$ with center $T x_{0}$ in the image space and a positive $\epsilon_{3} \leqq \epsilon_{2}$ such that $U \subset T^{*} S_{2}$ for all $\epsilon$ on $\left[0, \epsilon_{3}\right]$. The radius $\rho$ of $U$ is independent of $\epsilon$. 
Proof. We first note that $\left(T_{1}^{e}\right)^{-1}$ also satisfies a Lipschitz condition throughout $C$; specifically, for $0 \leqq \epsilon \leqq \epsilon_{2}$ and $y, y^{\prime} \in C$ we have

$$
\left\|||\left(T_{1}^{\epsilon}\right)^{-1} y-\left(T_{1}^{\epsilon}\right)^{-1} y^{\prime}\right\|\left|\leqq\left(1+B_{1}\right)\right||| y-y^{\prime}\|\| \text {. }
$$

For we have from (6.4) that

$$
\left(T_{1}^{\epsilon}\right)^{-1} y-\left(T_{1}^{\epsilon}\right)^{-1} y^{\prime}=y-y^{\prime}+\int_{0}^{1} k^{\epsilon}(\cdot, s)\left[y(s)-y^{\prime}(s)\right] d s,
$$

and (6.31) follows from (6.6).

Next we note that $\left(T_{3}^{\epsilon}\right)^{-1}$ also satisfies a Lipschitz condition throughout $C$; for $0 \leqq \epsilon \leqq \epsilon_{2}$ and $y, y^{\prime} \in C$, we have

$$
\left\|\left(T_{3}^{\epsilon}\right)^{-1} y-\left(T_{3}^{\epsilon}\right)^{-1} y^{\prime}\right\||| 2\left\||| y-y^{\prime}\right\| \| .
$$

For we have from (6.16) and (6.21),

$$
\begin{aligned}
\||| x-x^{\prime}|| \mid & =\left\||| T_{3}^{\epsilon} x-\Lambda_{3}^{\epsilon}(x \mid \cdot)-T_{3}^{\epsilon} x^{\prime}+\Lambda_{3}^{\epsilon}\left(x^{\prime} \mid \cdot\right)\right\| \| \\
& \leqq\left\||| T_{3}^{\epsilon} x-T_{3}^{\epsilon} x^{\prime}\left|\left\|+\frac{1}{2}\left|\left\|x-x^{\prime} \mid\right\|,\right.\right.\right.\right.
\end{aligned}
$$

and on transposing the second term of the right member and replacing $x$ and $x^{\prime}$ by $\left(T_{3}^{e}\right)^{-1} y$ and $\left(T_{3}^{e}\right)^{-1} y^{\prime}$, we obtain (6.32).

Now (6.31) and (6.32) obviously imply that for $0 \leqq \epsilon \leqq \epsilon_{2}$ and $y, y^{\prime} \in C$ we have the following Lipschitz condition for $\left(T_{1}^{e} T_{3}^{e}\right)^{-1}$ :

$$
\text { \| }\left|\left(T_{1}^{\epsilon} T_{3}^{\epsilon}\right)^{-1} y-\left(T_{1}^{\epsilon} T_{3}^{\epsilon}\right)^{-1} y^{\prime}\|\leqq \leqq \lambda\|\right| \mid y-y^{\prime}\|\|,
$$

where $\lambda=2\left(1+B_{1}\right)$. Hence $\left\|||\left(T_{1}^{e} T_{3}^{e}\right)^{-1} y-x_{0}\right\|||$ is a continuous function of $y$ on $C$, and the set where it is less than $r_{2}$ is open. Thus $T_{1}^{*} T_{3}^{*} S_{2}$ is open, and from (6.29) and (6.7), it follows that $T \mathcal{\epsilon}_{2}$ is open when $0 \leqq \epsilon \leqq \epsilon_{2}$.

Finally, let us choose $\rho=r_{2} / 2 \lambda$ and

$$
U=\left\{y \in C:\|\| y-T x_{0} \|<\rho\right\},
$$

and by (5.6.4) let us choose a positive $\epsilon_{3} \leqq \epsilon_{2}$ such that

$$
|| \mid \Lambda^{\epsilon}\left(x_{0} \mid \cdot\right)-\Lambda\left(x_{0} \mid \cdot\right) \|<\rho \quad \text { when } 0 \leqq \epsilon \leqq \epsilon_{3} .
$$

Then when $0 \leqq \epsilon \leqq \epsilon_{3}$ and $y \in U$, we have by (6.33), (6.28), and (6.7)

$$
\begin{aligned}
& \left\|\left(T_{1}^{\epsilon} T_{3}^{\epsilon}\right)^{-1} y-x_{0}\right\||| \leqq \lambda||\left|y-T_{1}^{\epsilon} T_{3}^{e} x_{0}\right| \| \\
& =\lambda\|\| y-T_{1}^{e} T_{2}^{e} x_{0}\|\|=\lambda\left\||| y-T^{\epsilon} x_{0}\right\| \mid \\
& \leqq \lambda\left\|y-T x_{0}\right\|\|+\lambda\||| T x_{0}-T^{e} x_{0}\|\| \\
& <\lambda \rho+\lambda||\left|\Lambda\left(x_{0} \mid \cdot\right)-\Lambda^{\epsilon}\left(x_{0} \mid \cdot\right) \|\right| \mid \leqq 2 \lambda \rho=r_{2} \text {. }
\end{aligned}
$$

Thus for $0 \leqq \epsilon \leqq \epsilon_{3}$, we have by (6.29) and (6.7) that 


$$
y \in U \rightarrow\left(T_{1}^{\epsilon} T_{3}^{\epsilon}\right)^{-1} y \in S_{2} \rightarrow y \in T_{1}^{\epsilon} T_{3}^{\epsilon} S_{2} \rightarrow y \in T_{1}^{\epsilon} T_{2}^{\epsilon} S_{2} \rightarrow y \in T^{\epsilon} S_{2}
$$

and the lemma is proved.

7. Proof of Theorem 3a. We now proceed with the proof of Theorem 3a, dividing the proof into a number of cases.

CASE I. For this case we make the following additional assumptions: $\Gamma=S$ $=S_{2}$; and $F(y)$ is bounded and continuous in the uniform topology and vanishes outside the sphere $U$ defined in Lemma 7.

To establish Case I, we first note that the conclusions of Lemmas 3-7 concerning $\Lambda^{\epsilon}, K^{\epsilon}$, and $D^{\epsilon}$ all hold; and Lemmas 3, 4, and 6 (including statement (6.3) of Lemma 6) imply that the hypotheses (and hence the conclusions) of Theorem A hold for the transformation $T^{\epsilon}$ in $S_{2}$ when $0<\epsilon \leqq \epsilon_{2}$. Thus we have that $T{ }^{\epsilon} S_{2}$ is Wiener measurable and that

$$
\int_{T^{\epsilon} S_{2}}^{w} F(y) d_{w} y=\int_{S_{2}}^{w} F\left(T^{\epsilon} x\right) e^{-\Phi^{\epsilon}(x)}\left|D^{\epsilon}(x)\right| d_{w} x,
$$

where

$$
\Phi^{\epsilon}(x)=\int_{0}^{1}\left[\frac{\partial}{\partial t} \Lambda^{\epsilon}(x \mid \epsilon)\right]^{2} d t+2 \int_{0}^{1}\left[\frac{\partial}{\partial t} \Lambda^{\epsilon}(x \mid t)\right] d x(t) .
$$

But by the assumption, $F(y)$ vanishes outside $U$ and by Lemma 7 we know that $U$ is contained in both $T{ }^{\epsilon} S_{2}$ and in $T S_{2}\left(=T^{0} S_{2}\right)$, for $0<\epsilon \leqq \epsilon_{3}$. Moreover $T S_{2}$ is Wiener measurable, since by Lemma 7 it is open. Thus for positive $\epsilon \leqq \epsilon_{3}$ we have

$$
\int_{T^{\epsilon} S_{2}}^{w} F(y) d_{w} y=\int_{u}^{w} F(y) d_{w} y=\int_{T S_{2}}^{w} F(y) d_{w} y,
$$

so that by (7.1),

$$
\int_{T S_{2}}^{w} F(y) d_{w} y=\int_{S_{2}}^{w} F\left(T^{\epsilon} x\right) e^{-\Phi^{\epsilon}(x)}\left|D^{\epsilon}(x)\right| d_{w} x .
$$

Let us now take limits in (7.3) as $\epsilon \rightarrow 0^{+}$. Since the left member is independent of $\epsilon$, it is unchanged, and we shall seek to show that the right member approaches the right member of (7.4) below, which will then be established.

$$
\int_{T S_{2}}^{w} F(y) d_{w} y=\int_{S_{2}}^{w} F(T x) e^{-\Phi(x)}|D(x)| d_{w} x
$$

where

$$
\Phi(x)=\int_{0}^{1}\left[\frac{\partial}{\partial t} \Lambda(x \mid t)\right]^{2} d t+2 \int_{0}^{1}\left[\frac{\partial}{\partial t} \Lambda(x \mid t)\right] d^{*} x(t) .
$$


To establish this, we note that $F\left(T^{\circ} x\right) \rightarrow F(T x)$ boundedly, since $F$ is bounded and continuous and (5.6.4) holds. Moreover $D^{\epsilon}(x) \rightarrow D(x)$ in $S_{2}$ by (5.6.5), and the approach is bounded since, by (5.4), $K^{\epsilon}$ is uniformly bounded. Thus (7.4) will be established and Case I will be proved when we show that

$$
\lim _{\epsilon \rightarrow 0^{+}} \Phi^{\epsilon}(x)=\Phi(x) \quad \text { for almost all } x \text { in } S_{2},
$$

and the limitand of the left member of (7.6) is bounded below.

To establish (7.6), we first note from (1.14.1) that for all $x$ in $S_{2}$

$$
\lim _{\epsilon \rightarrow 0^{+}} \int_{0}^{1}\left[\frac{\partial}{\partial t} \Lambda^{\epsilon}(x \mid t)\right]^{2} d t=\int_{0}^{1}\left[\frac{\partial}{\partial t} \Lambda(x \mid t)\right]^{2} d t ;
$$

and of course the limitand of the left member is bounded below. We next proceed to establish for almost all $x \in S_{2}$

$$
\lim _{\epsilon \rightarrow 0^{+}} \int_{0}^{1}\left[\frac{\partial}{\partial t} \Lambda^{\epsilon}(x \mid t)\right] d x(t)=\int_{0}^{1}\left[\frac{\partial}{\partial t} \Lambda(x \mid t)\right] d^{*} x(t),
$$

together with the fact that the limitand of the left member is bounded below. To do this, we must study the relationship between $[\partial \Lambda(x \mid t) / \partial t]_{\epsilon}$ and $\partial \Lambda^{e}(x \mid t) / \partial t$ or the equal quantity $\partial \Lambda_{\epsilon}(x \mid t) / \partial t$. Since differentiation and smoothing operators are commutative where they apply freely, these two expressions must be equal on $[0,1-\epsilon)$; but since for $t>1$ we must interpret $\Lambda(x \mid t)$ and $\Lambda_{t}(x \mid t)$ as $\Lambda(x \mid 1)$ and $\Lambda_{t}(x \mid 1)$ respectively in taking smoothings, the smoothing of the derivative exceeds the derivative of the smoothing by $(t+\epsilon-1) \epsilon^{-1} \Lambda_{t}\left(x_{\epsilon} \mid 1\right)$ on $(1-\epsilon]$. Thus we have for $t \in I, t \neq 1-\epsilon$,

$$
\left[\frac{\partial}{\partial t} \Lambda(x \mid t)\right]_{\epsilon}-\frac{\partial}{\partial t} \Lambda^{\epsilon}(x \mid t)=\frac{\max [t+\epsilon-1,0]}{\epsilon} \Lambda_{t}\left(x_{\epsilon} \mid 1\right),
$$

and

$$
\begin{aligned}
\int_{0}^{1}\{[ & \left.\left.\frac{\partial}{\partial t} \Lambda(x \mid t)\right]_{\epsilon}-\frac{\partial}{\partial t} \Lambda^{\epsilon}(x \mid t)\right\} d x(t) \\
& =\frac{1}{\epsilon} \int_{1-\epsilon}^{1}(t+\epsilon-1) d x(t) \Lambda_{t}\left(x_{\epsilon} \mid 1\right) \\
& =\left\{x(1)-\frac{1}{\epsilon} \int_{1-\epsilon}^{1} x(t) d t\right\} \Lambda_{t}\left(x_{\epsilon} \mid 1\right) .
\end{aligned}
$$

But it is clear from (1.14.1) and the continuity of $x(t)$ that the last member of (7.9) approaches zero as $\epsilon \rightarrow 0^{+}$; and hence (1.14.6) and (7.9) (together with the definition of a functional principal value, (1.10)) show that (7.8) holds for almost all $x$ in $S_{2}$. Finally (7.7) and (7.8) show that (7.6) holds almost everywhere in $S_{2}$, and (1.14.7) insures that the limitand on the left of 
(7.6) is bounded below. Thus (7.4) follows from (7.3) by bounded convergence, and Case I is established.

CASE II. For this case we make the assumption that $F(y)$ is the characteristic functional of an interval

$$
\begin{gathered}
J: \alpha_{j}<x\left(t_{j}\right)<\beta_{j} \\
\left(0<t_{1}<t_{2}<\cdots<t_{N} \leqq 1 ;-\infty \leqq \alpha_{j} \leqq \beta_{j} \leqq+\infty\right),
\end{gathered}
$$

and that $\Gamma=S=S_{3}$, where $S_{3}$ is an open sphere about $x_{0}$ of radius $r_{3}<r_{2}$ such that $T S_{3} \subset U$. The fact that such a sphere exists follows from the continuity of $T x$. (See (6.7), (6.15), and (6.2).)

In order to establish Case II, we apply Case I to the functional

$$
F_{\eta}(y)=\phi_{0, \eta}\left(\left\|\left(T_{1} T_{3}\right)^{-1} y-x_{0}\right\|\right) \prod_{j=1}^{N} \phi_{j, \eta}\left(y\left(t_{j}\right)\right)
$$

where $\phi_{j, \eta}(u)$ is a continuous "trapezoidal" function which equals zero outside the interval $\alpha_{j}<u<\beta_{j}$; equals unity on the interval

$$
\alpha_{j}+\eta \leqq u \leqq \beta_{j}-\eta
$$

and is linear on the remaining intervals; and for the case $j=0$ we take $\alpha_{0}=-\infty, \beta_{0}=r_{3}$. Since $\left(T_{1} T_{3}\right)^{-1}$ satisfies the Lipschitz condition (6.33) throughout $C, F_{\eta}(y)$ is continuous throughout $C$; and since $T^{-1} y=\left(T_{1} T_{3}\right)^{-1} y$ on $T S_{2}$ by (6.30) and (6.7), it follows that

$$
F_{\eta}(y)=\phi_{0, \eta}\left(\left\|\left|T^{-1} y-x_{0} \|\right| \mid\right) \prod_{j=1}^{N} \phi_{j, \eta}\left(y\left(t_{j}\right)\right) \quad \text { if } y \in T S_{2} .\right.
$$

Thus $F_{\eta}(y)$ vanishes outside $T S_{3}$, and consequently outside $U$. Hence the extra conditions of Case I are satisfied if we take its $\Gamma=S_{2}$, and we have (7.4) holding with $F$ replaced by $F_{\eta}$.

Now notice that $F_{\eta}(y)$ increases monotonically as $\eta$ decreases, and that

$$
\lim _{\eta \rightarrow 0+} F_{\eta}(y)=F(y) \chi_{T s_{3}}(y),
$$

where for any set $E, \chi_{E}(y)$ denotes the characteristic functional of $E$. Thus, by monotonic convergence, we may take the limit as $\eta \rightarrow 0^{+}$on both sides of (7.4) with $F_{\eta}$ replacing $F$, and we obtain

$$
\int_{T S_{3}}^{w} F(y) d_{w} y=\int_{S_{3}}^{w} F(T x) e^{-\Phi(x)}|D(x)| d_{w} x .
$$

Thus Case II is established; and in particular it has been established that $F(T x)$ is measurable on $S_{3}$, or that $S_{3} \cap T^{-1} J$ is measurable. We also call attention to the fact that a somewhat similar argument establishes the measurability of $T\left(J \cap S_{3}\right)$. For we note that the characteristic functional of this 
set is

$$
\lim _{\eta \rightarrow 0+} \phi_{0, \eta}\left(||\left|\left(T_{1} T_{3}\right)^{-1} y-x_{0} \|\right| \mid\right) \prod_{j=1}^{N} \phi_{j, \eta}\left[\left(T_{1} T_{3}\right)^{-1} y\left(t_{j}\right)\right],
$$

and hence is measurable.

CASE III. For this case we make the assumption that $F(y)$ is the characteristic functional of a measurable set $G$ in $C$. To establish this case we choose $S=S_{3}$, and we first consider the subcase in which $\Gamma=S$. Thus if $G$ is an interval, this subcase reduces to Case II, and is established. If $G$ is the union of a countable set of intervals, the theorem holds for each interval, and hence by monotone convergence for $G$. If $G$ is the intersection of a countable set of unions of countable sets of intervals, the theorem again holds by monotone convergence. If $G$ is a null set, it is a subset of a null set of the preceding type, and the theorem follows trivially. If $G$ is an unrestricted measurable set, it is the difference of sets of the two preceding types, and the result again follows trivially. Thus the subcase in which $\Gamma=S$ is established.

To establish Case III in general, we must show that for a measurable set $\Gamma \subset S$, the transformed set $T \Gamma$ is also measurable. But this was shown at the end of Case II for sets $\Gamma$ of the form $J \cap S$, where $J$ is an interval; and our theorem therefore holds for $\Gamma$ of this type, since we can apply the subcase to the functional

$$
\chi_{G \cap T(J \cap S)}(y)=F(y) \chi_{T(J \cap S)}(y) .
$$

The proof of Case III is completed by simply enlarging the class of sets $J$ from intervals to measurable sets as we $\operatorname{did}$ for $G$ in the subcase.

Case IV. The general case. We now enlarge the class of functionals from characteristic functionals of measurable sets (for which the theorem was proved in Case III) to general measurable functionals. This can be done in the usual way, extending first to simple functionals, then to non-negative measurable functionals, and then to general measurable functionals. Thus Theorem $3 \mathrm{a}$ is established.

8. The proof of Theorem 4a. In order to prove Theorem $4 \mathrm{a}$, let us first see what Theorem $3 a$ enables us to conclude. If $x_{0}$ is any point of our set $\Gamma$, then there exists a uniform neighborhood $S\left(x_{0}\right)$ contained in $S_{0}\left(x_{0}\right)$ such that the desired transformation formula holds for any Wiener measurable subset of $S\left(x_{0}\right)$; and the transformation $T$ takes this subset into a measurable set. Now let $x_{1}, x_{2}, \cdots$ be a countable set of points of $\Gamma$ whose neighborhoods $S\left(x_{1}\right), S\left(x_{2}\right), \cdots$ cover $\Gamma$. (Since $C$ is a separable metric space in the uniform topology, any covering can be reduced to a countable covering, and therefore such a set $\left\{x_{n}\right\}$ exists.) Then $\Gamma \subset \cup_{n=1}^{\infty} S\left(x_{n}\right)$, and if $\Gamma_{n}=\Gamma \cap S\left(x_{n}\right)$, we have $\Gamma=\bigcup_{n=1}^{\infty} \Gamma_{n}$. The desired transformation formula (1.15) applies to each set( $\left.{ }^{4}\right)$

(4) It is understood that $\bigcup_{k=1}^{0} \Gamma_{k}$ denotes the empty set. 
$\Gamma_{n}-\bigcup_{k=1}^{n-1} \Gamma_{k}(n=1,2, \cdots)$, and the transform of each of these sets is measurable. Since the Wiener integral is completely additive for non-negative integrands, we may sum over $n$ and thus obtain the measurability of $T \Gamma$ and the validity of (1.15) for non-negative $F(y)$. The extension to general $F(y)$ is immediate, and Theorem $4 \mathrm{a}$ is proved.

9. The proofs of Theorems 3 and 4 . We shall show that Theorem 3 follows from Theorem $4 \mathrm{a}$ in the same way that Theorem 3a follows from Theorem A. We first note that under the hypotheses of Theorem 3, Lemmas 1-7 still hold, except that in Lemma 4 we can no longer conclude that $K^{\epsilon}$ satisfies condition (3.1.8). In particular, we rote from Lemma 6 that $T^{\epsilon}$ takes $S_{2}$ into $T^{\epsilon} S_{2}$ in a 1-to-1 way. We now take $S_{2}$ to be the $\Gamma$ of Theorem 4 a.

We next verify that when $0<\epsilon<\epsilon_{3}, \Lambda^{\epsilon}$ and $K^{\epsilon}$ satisfy the hypotheses of Theorem 4 a; i.e., that they satisfy the hypotheses of Theorem 3 as well as Condition (4.1) in a neighborhood $S_{0}=S_{0}\left(x_{0}^{\prime}\right)$ of each point $x_{0}^{\prime}$ of $\Gamma \equiv S_{2}$. By Lemma 3 we know that $\Lambda^{e}$ is of smooth variation in some Hilbert neighborhood $H_{\epsilon}\left(x_{0}^{\prime}\right)$ (and hence also in some uniform neighborhood $S_{\epsilon}\left(x_{0}^{\prime}\right)$ of each point $x_{0}^{\prime}$ of $\left.S_{2}\right)$, and that (5.2) holds. Clearly we can choose the $S_{\epsilon}\left(x_{0}^{\prime}\right)$ so that each of these spheres is contained in the open sphere $S_{2}$, and we then take $S_{\epsilon}\left(x_{0}^{\prime}\right)$ to be the desired $S_{0}\left(x_{0}^{\prime}\right)$. By Lemma 4 and by (6.3) of Lemma 6 , we know that (3.1.1)-(3.1.7) hold in $H_{\epsilon}\left(x_{0}^{\prime}\right)$ for $x_{0}^{\prime} \in S_{2}$, and hence that (1.14.2)-(1.14.5) hold for $\Lambda^{\epsilon}$ and $K^{\epsilon}$ in $S_{\epsilon}\left(x_{0}^{\prime}\right)$ whenever $x_{0}^{\prime} \in S_{2}$. It is easy to see from the definition of $\Lambda^{\bullet}$ that it satisfies (1.14.1) in $S\left(x_{0}^{\prime}\right)$, using the fact that $\Lambda$ satisfies (1.14.1) by hypothesis.

Next we show that $\Lambda^{\epsilon}$ satisfies (1.14.6) in $S_{\epsilon}\left(x_{0}^{\prime}\right)$. We note that $\partial \Lambda^{\epsilon}(x \mid t) / \partial t$ satisfies the hypotheses of Theorem 1 , since it equals $\left[\Lambda\left(x_{\epsilon} \mid t+\epsilon\right)\right.$ $\left.-\Lambda\left(x_{\epsilon} \mid t\right)\right] / \epsilon$ and

$$
\begin{aligned}
\operatorname{var} & {\left[\frac{\partial}{\partial t} \Lambda^{\epsilon}(y \mid t)-\frac{\partial}{\partial t} \Lambda^{\epsilon}(x \mid t)\right] } \\
& =\frac{1}{\epsilon} \int_{0}^{1}\left|\Lambda_{t}\left(y_{\epsilon} \mid t+\epsilon\right)-\Lambda_{t}\left(x_{\epsilon} \mid t+\epsilon\right)-\Lambda_{t}\left(y_{\epsilon} \mid t\right)+\Lambda_{t}\left(x_{\epsilon} \mid t\right)\right| d t .
\end{aligned}
$$

Since, by (1.14.1), $\Lambda_{t}(x \mid t)$ is continuous in $(x, t)$ on $S_{0} \otimes I$ it follows that it is continuous in $x$ uniformly with respect to $t$, and hence that the right member of (9.1) approaches zero as $y \rightarrow x$. (Actually the right- and left-hand derivatives of $\Lambda(x \mid t)$ may be different at $t=1$, but this causes no difficulty.) We thus have in $S_{\epsilon}\left(x_{0}^{\prime}\right)$ the existence of the functional principal value of the Stieltjes integral of $\partial \Lambda^{\epsilon} / \partial t$ and the equality

$$
\int_{0}^{1} \frac{\partial}{\partial t} \Lambda^{\epsilon}(x \mid t) d^{*} x(t)=\int_{0}^{1} \frac{\partial}{\partial t} \Lambda^{\epsilon}(x \mid t) d x(t) .
$$

Thus $\Lambda^{e}$ satisfies (1.14.6) in $S_{\epsilon}\left(x_{0}^{\prime}\right)$.

To show that $\Lambda^{\epsilon}$ satisfies (1.14.7) in $S_{\epsilon}\left(x_{0}^{\prime}\right)$, we must show that for each 
fixed $\epsilon$, on $0<\epsilon \leqq \epsilon_{3}$, and for some positive number $\epsilon_{1}$ (which can be conveniently chosen to be the $\epsilon_{1}$ defined in Lemma 3),

$$
\int_{0}^{1} \frac{\partial}{\partial t}\left\{\left[\Lambda^{\epsilon}(x \mid t)\right]_{\epsilon^{\prime}}\right\} d x(t)
$$

is bounded below in $x$ and $\epsilon^{\prime}$ on $S_{\epsilon}\left(x_{0}^{\prime}\right) \otimes\left(0, \epsilon_{1}\right)$. But clearly the expression (9.3) can be written

$$
\frac{1}{\epsilon^{\prime}} \int_{0}^{1}\left\{\Lambda_{\epsilon}\left(x_{\epsilon^{\prime}} \mid t+\epsilon^{\prime}\right)-\Lambda_{\epsilon}\left(x_{\epsilon^{\prime}} \mid t\right)\right\} d x(t),
$$

where our convention requires that the first term in braces be $\Lambda_{\epsilon}\left(x_{\epsilon^{\prime}} \mid 1\right)$ when $t+\epsilon^{\prime}>1$. Integrating by parts, we find that (9.3) is equal to

$$
\begin{aligned}
& -\frac{1}{\epsilon^{\prime}} \int_{0}^{1} x(t) \frac{\partial}{\partial t}\left\{\Lambda_{\epsilon}\left(x_{\epsilon^{\prime}} \mid t+\epsilon^{\prime}\right)-\Lambda_{\epsilon}\left(x_{\epsilon^{\prime}} \mid t\right)\right\} d t \\
& \quad=-\frac{1}{\epsilon^{\prime}} \int_{0}^{1+\epsilon^{\prime}} x\left(t-\epsilon^{\prime}\right) \frac{\partial}{\partial t} \Lambda_{\epsilon}\left(x_{\epsilon^{\prime}} \mid t\right) d t+\frac{1}{\epsilon^{\prime}} \int_{0}^{1} x(t) \frac{\partial}{\partial t} \Lambda_{\epsilon}\left(x_{\epsilon^{\prime}} \mid t\right) d t \\
& \quad=\int_{0}^{1} \frac{\partial}{\partial t} \Lambda_{\epsilon}\left(x_{\epsilon^{\prime}} \mid t\right) d x_{\epsilon^{\prime}}(t) .
\end{aligned}
$$

Thus expression (9.3) is simply the expression in (1.14.7) with $x$ replaced by $x_{\epsilon^{\prime}}$. Thus by (1.14.7) we have that (9.3) is bounded below in $\epsilon, \epsilon^{\prime}$, and $x$ if $0<\epsilon<\epsilon_{0}$ and $x_{\epsilon^{\prime}} \in S_{0}$. But now if $0<\epsilon^{\prime}<\epsilon_{1}$, we have by the first paragraph of the proof of Lemma 3 that $\left\|\left|x_{0, e^{\prime}}-x_{0}\right|\right\|<r_{0} / 3$, and hence that if $x \in S_{1}$, $\left\||| x_{\epsilon^{\prime}}-x_{0}\right\|||<2 r_{0} / 3$ and $x_{\epsilon^{\prime}} \in S_{0}$. Also we have $S_{\epsilon}\left(x_{0}^{\prime}\right) \subset S_{2} \subset S_{1}$, so $(9.3)$ is bounded below in the desired region; i.e., when $0<\epsilon \leqq \epsilon_{3}, 0<\epsilon^{\prime}<\epsilon_{1}, x \in S_{\epsilon}\left(x_{0}^{\prime}\right)$, and $x_{0}^{\prime} \in S_{2}$. Hence $\Lambda^{e}$ satisfies (1.14.7) in the proper sense.

Finally, we note that (4.1) is satisfied by $K^{\epsilon}$ in $S_{\epsilon}\left(x_{0}^{\prime}\right) \otimes I$ when $0<\epsilon \leqq \epsilon_{3}$. This follows readily from (1.14.4) and (5.2), since

$$
\frac{\partial}{\partial t} K^{\epsilon}(x \mid t, s)=\frac{1}{\epsilon^{2}} \int_{s}^{\min (s+\epsilon, 1)}\left\{K\left(x_{\epsilon} \mid t+\epsilon, u\right)-K\left(x_{\epsilon} \mid t, u\right)\right\} d u
$$

and

$$
\operatorname{var}_{t}\left[K^{e}(x \mid t, s)\right]=\int_{0}^{1}\left|\frac{\partial}{\partial t} K^{e}(x \mid t, s)\right| d t .
$$

Thus the hypotheses of Theorem 4a are all satisfied, and we may employ it in proving Theorem 3 exactly as Theorem A was used in proving Theorem $3 \mathrm{a}$. The conclusions of Theorems A and $4 \mathrm{a}$ are now identical because of (9.2), and the extra hypothesis (4.1) of Theorem 3a is nowhere else used in the proof. Thus Theorem 3 is established, 
Theorem 4 follows from Theorem 3 exactly as Theorem 4a follows from Theorem $3 \mathrm{a}$, and is therefore also established.

10. Conclusion. We close this paper by pointing out that Theorems 3 and 4 are strict generalizations of Theorems IV and V of [2]. This can easily be verified if the reader keeps in mind (1.8) of [2], as well as Theorem 1 and formula (7.9) of this paper.

\section{REFERENCES}

1. R. H. Cameron and W. T. Martin, Transformations of Wiener integrals under a general class of linear transformations, Trans. Amer. Math. Soc. vol. 58 (1945) pp. 184-219.

2. - The transformation of Wiener integrals by nonlinear transformation, Trans. Amer. Math. Soc. vol. 66 (1949) pp. 253-283.

3. Paul Levy, Leçons d'analyse fonctionnelle, Paris, Gauthier-Villars, 1922.

UNIVERSITY OF MINNESOTA, MinnEAPOLIS, MinN. 